\title{
PaperCP: Exploring the Integration of Physical and Digital Affordances for Active Learning
}

\author{
Chunyuan Liao ${ }^{1}$, François Guimbretière ${ }^{1}$, Richard Anderson ${ }^{2}$, Natalie Linnell ${ }^{2}$, \\ Craig Prince ${ }^{2}$, and Valentin Razmov ${ }^{2}$ \\ ${ }^{1}$ Dept. of Computer Science, Univ. of Maryland, College Park, U.S.A \\ ${ }^{2}$ Dept. of Computer Science \& Engineering, Univ. of Washington, Seattle, U.S.A \\ \{liaomay, francois\}@cs.umd.edu; \\ \{anderson, linnell, cmprince, valentin\}@cs.washington.edu
}

\begin{abstract}
Active Learning in the classroom domain presents an interesting case for integrating physical and digital affordances. Traditional physical handouts and transparencies are giving way to new digital slides and PCs, but the fully digital systems still lag behind the physical artifacts in many aspects such as readability and tangibility. To better understand the interplay between physical and digital affordances in this domain, we developed PaperCP, a paper-based interface for a Tablet PC-based classroom interaction system (Classroom Presenter), and deployed it in an actual university course. This paper reports on an exploratory experiment studying the use of the system in a real-world scenario. The experiment confirms the feasibility of the paper interface in supporting student-instructor communication for Active Learning. We also discuss the challenges associated with creating a physical interface such as print layout, the use of pen gestures, and logistical issues.
\end{abstract}

Keywords: Active Learning, Affordances, Paper-based Interface, Physical Interface, Tablet PC.

\section{Introduction}

Active Learning refers to augmenting the traditional lecture with student-participation activities such as brainstorming, quizzing, and polling. Also vital to Active Learning is sharing (e.g., displaying) student responses as part of a lecture. Because of this twoway instructor-student communication, Active Learning increases student engagement, helps with the construction of knowledge, and improves the level of understanding of students, as well as the instructors' awareness of it [25].

Designing a system to support Active Learning is challenging because of the tension between traditional physical interfaces and newer electronic ones. For example, traditional printouts and transparencies are easy to read and write on, convenient to navigate, and easy to manipulate by hand. But the manual distribution, collection, summarization, and display of the physical artifacts is often inefficient and distracting. To address this issue, fully digital systems have been developed [3, 10, 24, 29]. For instance, Classroom Presenter (CP) [3], a digital Active Learning system, allows the instructor to deliver slides and gather student responses wirelessly via networked 
pen-based Tablet PCs. Despite the digital solutions' advantages in data transfer and archiving, some drawbacks are associated with these systems: a degraded reading and writing experience due to limited screen size and screen resolution, the cost of the devices, and the limitations imposed by battery

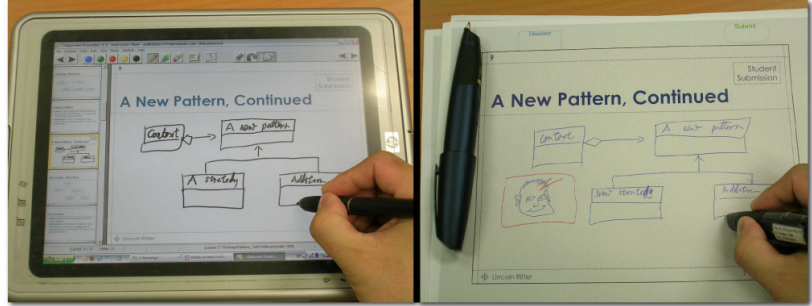

Fig. 1. (Left) The original Tablet PC interface of a digital Active Learning infrastructure. (Right) The new equivalent interface, PaperCP, based on Anoto technology, which consists of Bluetooth digital pens and printouts. life. To ease the tension between physical and digital affordances, a natural solution is to integrate them to create a better overall user experience.

In this paper, we investigate how to combine the advantages of physical artifacts like paper with the convenience of an electronic communication and archiving infrastructure. Specifically, based on a communication model for Active Learning, we propose a new Anoto [5]-based paper interface, PaperCP (Paper Classroom Presenter) (Figure 1), for Classroom Presenter, aimed at addressing the interaction and costbenefit problems of the fully digital system. Our physical interface allows students to use Anoto-enabled slide printouts as an input interface, so that users can still enjoy the inherent advantages of paper. Using a digital pen, students can write directly on the handouts and can electronically submit their handwritten notes to the instructor, thereby maintaining two-way communication with the instructor. Furthermore, the compatibility of paper and digital interfaces allows multiple heterogeneous interfaces (i.e., paper and Tablet PC) to be simultaneously deployed with our system, so that users can choose which system to use for a given Active Learning activity.

To evaluate this system, we deployed it during four regular class sessions of an actual Software Engineering course at the University of Washington. Using qualitative in-class observations in addition to quantitative results from questionnaires and user logs, our study confirmed the feasibility of implementing and deploying PaperCP in a real-use scenario. The study also revealed that the choice of a print layout and digital pen configuration has a large impact on the perceived tangibility advantage of the physical interface. Finally, our experiences provide insight into designing new interfaces that combine paper and digital affordances.

\section{Related Work}

\subsection{Computer-Supported Teaching and Learning}

Technological support for teaching has been informed by the educational literature on the difficulties of engaging students with traditional university-style lectures [7, 9]. Active Learning [16] was proposed to address such issues by promoting in-class 
student involvement through activities, e.g., in Classtalk [10], and actively seeking feedback on the level of student learning [4].

The idea behind much of the work in classroom technology has been to enhance or offload certain activities, so that students and instructors can be more effective in the classroom. Opportunities for this include capturing the classroom experience to reduce note taking demands in Classroom 2000 [1], creating new communication channels for student-student in-class interaction in LiveNotes [17] and student-instructor communication in Classtalk [10], ActiveClass [24], and Classroom Presenter [3].

Specifically, Classroom Presenter (CP) supports sharing ink-based artifacts between students and the instructor in real-time by using networked Tablet PCs. Other similar systems include Debbie (now DyKnow) [11] and Ubiquitous Presenter [29]. Classroom Response Systems (also known as clickers) [10, 21] take a different approach, aggregating student information, as opposed to providing rich individual responses. Our system is built on top of the $\mathrm{CP}$ infrastructure, supporting the same real time student-instructor communication with a new paper interface.

The communication in Active Learning could also be non-real-time, e.g. without a fixed time relation between the actions of students and the instructor. For instance, ActiveClass [24] allows students to use PDAs to deliver asynchronous feedback to the instructor via web pages; Classroom Feedback Systems [2] are similar.

\subsection{Paper-Computer Integration}

There are many systems in the literature detailing paper-computer integration. Among them are DigitalDesk [28], A-Book [20] and PaperWindows [15], all of which use augmenting devices, such as overhead projectors, graphics tablets, and cameras to overlap digital and paper display content. Despite their high display fidelity and powerful digital functions, the augmenting devices' lack of portability limits these systems' paper affordances.

New Anoto technology [5] allows handwriting capture on paper with highly portable digital pens. Based on this, PADD [13] supports mapping handwriting from printouts to the corresponding digital pages. Using the PADD infrastructure, PapierCraft [18] proposes a paper command system by using paper documents as a proxy to their digital copies, and mapping pen gesture commands on paper to corresponding digital manipulations. ButterflyNet [30] employs PapierCraft gestures to help field biologists organize and collect multimedia data centered on paper. The work presented in this paper borrows the idea of a "paper proxy", but targets the real-time student-instructor communication in Active Learning.

In the literature, similar real-time paper-digital interaction applications include $\mathrm{Pa}$ perPoint [27]. Based also on Anoto technology, PaperPoint helps a presenter to annotate and select digital slides to show via printouts. In contrast, PaperCP focuses on instructor-student communication during in-class activities. Designed specifically for presentation practices, Palette [22] uses paper index cards to organize and choose digital slides to display, but it does not support digital editing on paper and is less general than PaperPoint. 


\section{A Communication Model of Active Learning}

Active Learning involves students performing activities in the classroom and communicating with other students and the instructor. Here we focus on the studentinstructor interactions, which can be characterized by the following communication model.

As illustrated in Fig. 2, there are two parties in the model, the instructor and the students. The instructor navigates through slides, presenting prepared lecture material or showing student artifacts, perhaps adding on-the-fly comments or sketches according to the students' understanding level. Each student follows the

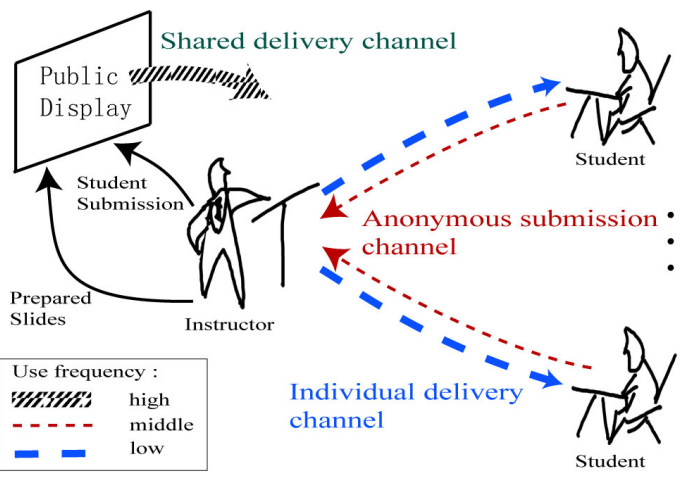

Fig. 2. A communication model of Active Learning. It consists of three channels denoted by dashed lines, whose thickness corresponds to the amount of data transmitted over each specific channel.

presentation; navigating and annotating his/her own copy of the lecture materials, as well as taking notes.

Active Learning is achieved by the two parties via three main communication channels (Figure 2): First, the instructor uses the individual delivery channel to distribute activities and lecture materials to individual students, e.g., traditional paper handouts, transparencies, or digital PowerPoint slides. These materials are relatively static through the course of the class and are intended for the students' personal use. The distribution typically occurs just once, at the beginning of a lecture. The channel's digital implementation is highly efficient. In comparison, delivering physical handouts before class is a burden, but will not severely affect what happens during the class time.

At certain points during the lecture, the instructor announces prepared activities to involve students in thinking and presenting ideas, such as drawing workflow diagrams, ranking key factors, selecting a multiple choice answer, or writing snippets of source code. Then, students use the anonymous submission channel to anonymously submit to the instructor answers or artifacts that they dynamically create during class. This channel is often accessed several times during a class session. Compared to handing in physical artifacts, electronic submission is much more efficient. It is important to note that the amount of data (e.g., brief comments, ranking, polls, as well as rich responses like graphs or drawings) traveling from the students to the instructor is usually smaller than in the reverse direction. This makes it possible to use a simple student interface with only inking functions to support Active Learning. The support for anonymous artifact submission is important for encouraging shyer students to take part in classroom activities too. 
After collecting student submissions, the instructor uses the shared delivery channel, usually a public display, for showing selected student-submitted artifacts. She may comment on the student answers, lead discussion on the open questions, and elicit further activities. Thus, the students not only receive feedback about their level of understanding, but are also encouraged to think more deeply about the lecture material. This channel is the dominant channel for class-wide presentation and is the focus of classroom discussions. The implementation of this channel increasingly employs digital projectors, which take the place of traditional overhead projectors or whiteboards, and accordingly student submissions need to have some electronic form to be displayed and discussed on the digital projectors.

\section{Classroom Presenter: A System Supporting the Model}

The PaperCP system is an extension of the Classroom Presenter system (Fig. 3), which employs wirelessly connected Tablet PCs for the two-way instructor-student communication. The instructor Tablet PC acts as a server, while the student Tablet PCs are clients. The individual delivery channel is implemented with IP multicast, through which all slides of a lecture can be efficiently delivered from the server to each student device at the beginning of the class. When the instructor reaches a slide with a prepared question, each student writes a response directly on their Tablet PC and digitally submits it via point-to-point connections to the instructor station. This is the anonymous submission channel. In addition, a public display conwwwnected to the instructor station is used as the shared delivery channel, which the instructor uses to display selected student submissions and offer her comments.

Classroom Presenter highlights the advantages of digital affordances:

- Efficiency: It makes instructor-student communication less distracting.

- Flexibility: Instructors can edit slides or student-submissions on-the-fly.

- Compatibility with existing practices using a data projector and digital slides.

However, there are drawbacks of the Tablet PC interface in terms of the interaction experience and the cost-benefit alignment:

- Tablet PCs are still relatively inferior to paper in terms of readability, writability, tangibility, and social acceptance.

- Computers can be distracting, as students may be tempted to use other unrelated applications (e.g., instant messaging or email) in class.

- Taking notes on a Tablet PC may be incompatible with some students' existing paper-based note taking styles.

- The cost of a Tablet PC may be too high for some students to own one.

In contrast to the Tablet PC interface, traditional physical printouts and transparencies are easy to read and write on, flexible in spatial layout, readily manipulated by hand, and relatively cheap. We explore how to integrate these advantages of physical interfaces into the highly efficient communication infrastructure of Classroom Presenter, in order to achieve a better balance between physical and digital affordances. 


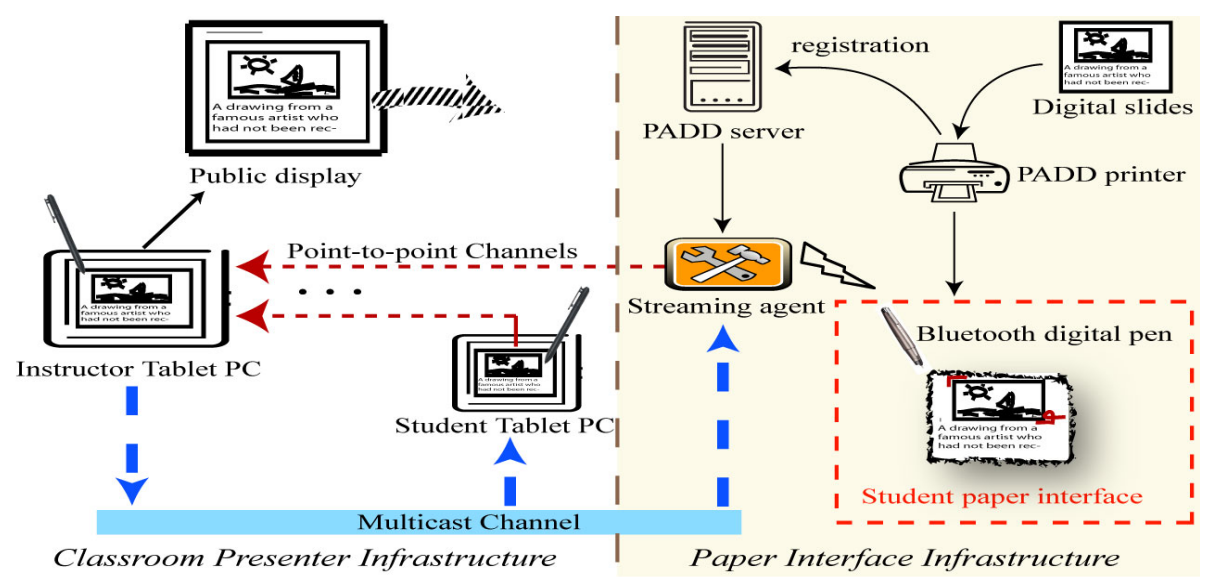

Fig. 3. Architecture of PaperCP system: an integration of the Classroom Presenter Infrastructure (left area) and the Paper Interface Infrastructure (right area). The three communication channels are denoted by dashed arrowed lines. The system supports the concurrent use of the student Tablet PC interface and the paper interface.

\section{Designing a Paper-Based Interface for Classroom Presenter}

The following principles guided our design:

- Paper affordances. Constraints on paper-based note-taking or annotating should be minimized since the paper affordances are the key to overcoming the limitations of the digital interface.

- Efficient communication. The paper interface should support efficient student submissions, a key enabling aspect of Active Learning communication.

- Compatibility. The paper-based interface should be deployable alongside a Tablet PC interface for flexibility in choosing and comparing interfaces.

- Realistic deployment. The implementation should be suitable for real classroom deployment, so that realistic user experiences can be observed.

Based on these principles, we first examined the Active Learning communication media according to the characteristics of relevant interactions.

For instructor interactions, the purely digital interface is believed to work best. First, the instructor needs an interface that allows her to review and selectively display digital student submissions that are dynamically created in class, which is impossible with a purely paper interface like PaperPoint [27]. Although an additional computer interface could be used for this task, frequent switching between paper and computer interfaces may be inconvenient and distracting to the instructor.

For student interactions, however, a paper-based interface is preferred because of its better trade-off between physical and digital affordances. First, paper possesses good interaction advantages as discussed above and a digital pen is much cheaper than a Tablet PC. Second, as used in PaperPoint [27], the new Bluetooth digital pens make it possible to capture and submit students' handwriting on paper in real time. Thus, a paper interface for digital submission is technically possible. 
As a result, we devised an architecture that mixes paper and computer components (Fig. 3). The instructor interface remains unchanged, but the student interface can be implemented either on paper (the part in the dashed box in the middle of Fig. 3) or on a Tablet PC. Both implementations share the same underlying Classroom Presenter infrastructure. The paper interface consists of only Bluetooth digital pens and slides printed on Anoto paper Fig. 4). Using digital pens, students can annotate the handouts or take notes as if using a normal pen and paper, and, more importantly, they can issue commands to delete or submit specific notes on paper via the underlying electronic communication channels.

\subsection{Student Note-taking and Submission}

The use of digital pens and printed slides is fully compatible with existing pen-and-paper note-taking practices. Students' writing on the printout is automatically captured by the digital pen. However, due to the static nature of printed content, we need special mechanisms to implement student submission, equivalent to that of the Tablet $\mathrm{PC}$ interface.

In the simplest case, the instructor uses a dedicated slide to solicit a submission from students, and the written artifacts on the slide are expected to be submitted. In such a case, students can submit their writing by simply ticking

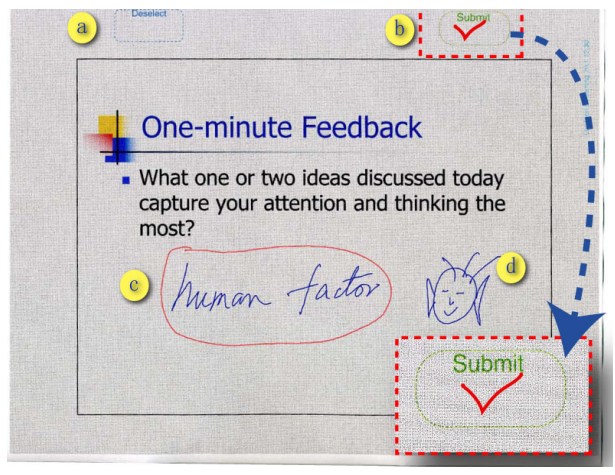

Fig. 4. A paper interface. (a) printed button "deselect". (b) printed button "submit". The inset illustrates a button ticking mark. (c) lasso selection to submit. (d) personal doodling, not for submission. Further, the area within the frame is "public" and the rest is "private". (i.e., drawing a check mark as illustrated in Fig. 4) the printed button labeled "submit" on the handouts. All digital notes captured from the slide are immediately sent to the instructor's computer. Our experiments show that such button clicking is very robust and easy to use.

However, when personal notes and activity answers occur in the same slide, we need to distinguish the public notes, which the student is willing to show to the class, from the private ones, which are for the student's private use (e.g., personal comments or doodles like (d) in Fig. 4). Similar privacy issues are discussed in systems such as Stitching [14], which supports varying cross-Tablet PC interaction patterns according to the collaboration levels of co-located users. PaperCP focuses on sharing notes with all class members via the public display, and thus presents as small a cognitive footprint as possible.

\subsection{Advanced Features}

While the Tablet PC interface provides dynamic visual feedback for distinguishing notes, e.g., by changing color of selected strokes, it is impossible for a paper-only interface to take the same approach. Instead, we examined three different methods for 
public-private note selection, namely spatial differentiation, pen-switching differentiation, and gesture differentiation. The adopted strategy was to combine spatial and gesture differentiation.

Spatial differentiation. The idea was borrowed from systems like SharedNotes [12] in which a specific region within a slide is defined as a "public" area and the rest of the slide is a "private" area. In our case, only notes within the public area (the area within the frame, see Fig. 4) may be shown on the public display. This design is intuitive and robust, but it prevents users from writing personal comments near pertinent information in the public area. Furthermore, users must determine a priori what to submit and what not to submit, which is not compatible with the typical user experience. Thus we turn to pen-based strategies.

Pen-switching differentiation. Another strategy is to use two pens: one for "public" notes and the other for "private" notes. Although this method can overcome the spatial constraint, our pilot test showed that this method also suffered from the a priori decision problem, and changing the type of a written stroke is often awkward. Furthermore, frequently switching pens turns out to be a non-trivial extra burden for users. So, we excluded this design.

Gesture differentiation. Here we borrow the ink/gesture approach of PapierCraft [18], a gesture-based command system for paper interfaces. In this approach, ink strokes are used to add notes or annotations at any location, and gestures strokes to issue commands like selecting ink strokes for submission. This method provides good spatial flexibility and avoids the a priori decision problem.

For distinguishing between ink and gesture strokes, our interface takes an explicit mode-based approach similar to PapierCraft's. Due to the lack of a convenient modeswitch button on the digital pen, we decided to use two pens, one for ink strokes and the other for gesture strokes. This two-pen configuration is far less problematic than that of the pen-switching differentiation due to the lower frequency of changing pens: users can first write free-form notes with the ink pen then select a subset of the content for submission with the gesture pen. Note that at this point the users can change their minds about any previously written strokes.

For easiness-flexibility tradeoff, we opted to combine the spatial and gesture differentiation methods as follows. If there is no selection gesture in the "public" area, all strokes in public area are sent, otherwise only the selected strokes are submitted. To further reduce the burden of switching pens, we allow submission with either pen. Thus, if a student uses only the simple spatial differentiation, she can keep using the ink pen for both writing and ticking the submission button.

Deleting unwanted content is another important issue. In many cases, simply crossing out unwanted strokes may be enough, but sometimes students want to remove certain writing from the digital record for neatness or privacy. To this end, PaperCP supports two deleting gestures, a zigzag, which removes strokes crossed by the gesture, and a lasso with zigzag inside, which deletes strokes inside the lasso. Of course, without physical erasing, the digitally deleted strokes will still remain on the printout. For the sake of simplicity, we have not implemented "undo" for stroke deletion. 


\subsection{System Implementation}

Fig. 3 illustrates the system architecture, in which the Streaming Agent plays a vital role in bridging the gap between the paper interface and the digital infrastructure. The agent receives handwriting captured by the digital pen via a Bluetooth connection, and contacts the PADD server for physical-to-digital mapping information, which was registered when the printouts were generated (refer to [13] for details). With the mapping information, the agent translates the strokes from physical page coordinates into a digital counterpart and then processes the data in the digital domain. Much like a standard student $\mathrm{CP}$ client, the agent communicates with the instructor $\mathrm{CP}$ station to receive broadcast slides and submit student input over a point-to-point channel. Thus, the new system scales similarly as the original Classroom Presenter [3] system, which can usually handle a classroom of about 30 student tablets.

The streaming agent implements the exactly same communication protocols as the original student $\mathrm{CP}$, so the paper-based interface is completely transparent to the rest of the $\mathrm{CP}$ infrastructure. One agent is needed for each paper interface user, and we use one PC near a user to run only one agent for simplicity. For larger classes and to minimize deployment costs, it is desirable to employ a Bluetooth infrastructure with multiple access points [8] distributed in the classroom, so that all digital pens are within Bluetooth signal range and multiple agents can share computing resources on one host computer.

\section{Exploratory User Study}

To examine the feasibility of our new paper interface and to explore possible design issues, we conducted an exploratory user study using PaperCP alongside the original Tablet PC-based system. Specifically, we focused on the following aspects of the system:

- Student-instructor communication: whether or not PaperCP can effectively support student-instructor communication for Active Learning.

- Interface integration: whether or not the paper interface can be naturally and efficiently integrated into Active Learning with little disruption.

- Gesture commands: whether or not the gesture operations on the paper interface can achieve the designed functionality.

\subsection{Experiment Setting}

The general goal of the experiment was to get a real sense about how a paperinterface works. We ran the experiment in a real-life scenario, instead of a lab setting, for a more realistic evaluation. During our evaluation, we changed as little as possible the instructor's lecture material, teaching style, and schedule, and did not force students to do any special actions. Finally, experiment data logging was done in the background without interference with participants and strictly kept anonymous. 
We deployed the system in an undergraduate Software Engineering class at the University of Washington. A 20-minute training session was first conducted to allow all students to try out the new paper interface and to answer questions. Students were asked to write their answers on handouts and submit them to the instructor, who then showed the submissions on a public display. Subsequently, we conducted four formal experimental sessions (each lasting 60 minutes) in regular classes on four days within a period of two weeks. During the sessions, the instructor used the Classroom Presenter infrastructure to present slides, collect student submission, and conduct in-class discussion as is usual for the course. The students, our participants, used either the Tablet PC interface or the paper counterpart for the Active Learning interactions.

To limit the variance introduced by different subjects and lecture topics in each class session, half of the students used the paper interface and the other half used the computer interface. Each student alternated between the two interfaces across sessions. At the end of each session, every student was asked to fill out a very brief questionnaire about the interface that he/she used during that session. After the final (fourth) session, they were asked to answer an overall comparison questionnaire.

For qualitative measurements, we used Likert-scale questionnaires about the users' perceived difficulty level of specific actions with an interface, such as note-taking, submission, and erasing. For quantitative measurements, we instrumented the two interfaces, and logged important events such as pen property changes, strokes, gestures, slide navigation, and submission.

\subsection{Apparatus and Participants}

During an experiment session, each participant was given a PaperCP interface or a Tablet PC running Classroom Presenter. In the current PaperCP implementation, we used a Tablet PC to run the Bluetooth data processing program (see section 5.3) for each participant, but it was placed several feet away from the participant, to minimize distraction. To reduce the user experience variance caused by the different size and layout of slides on the different interfaces, we intentionally printed one slide on one letter-sized sheet of paper, and made it roughly the size of its digital counterpart on a Tablet PC. For better navigation flexibility, we did not staple the handout pages.

All eight registered students for the class participated in the user study without pay. According to the background survey, two of the participants use laptops and another participant uses a Tablet PC to take notes occasionally. Seven participants frequently use normal pen and paper for notes. None of them had ever used a digital pen, but all of them had had experience with Classroom Presenter in previous courses.

\subsection{Experiment Results and Discussion}

We report the user experiences and lessons learned from the experiment with respect to our axes of evaluation: Student-instructor communication, Interface integration, and Gesture commands. Due to the small number of participants and high variability in the data, we focus here on qualitative rather than statistical analysis of the collected data. 


\subsubsection{Student-Instructor Communication}

There were 11 class activities in total during the four experimental sessions, or about 3 per session. The 11 class activities covered a wide range of question types, including drawing diagrams, ranking items, brainstorming answers to open-ended questions, commenting source code, drawing curves, and so on.

Fig. 5 illustrates such a digital student submission from the paper interface, in which the instructor added the comments in green during the public discussion.

Generally, all 11 activities went smoothly without any show-stopping technical problems. The paper interface

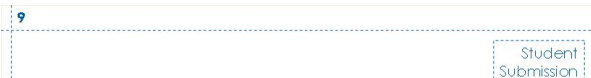

A New Pattern, Continued submission

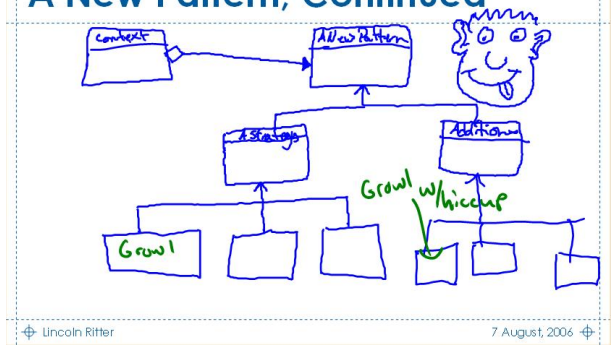

Fig. 5. An example of a student submission from the paper interface. The green strokes are the instructor's comments. was well-received by the students. In terms of the workflow, both interfaces successfully sent student submissions in real time. The paper interface was used by students as actively as the Tablet PC interface. According to our log data, the instructor received 33 submissions from paper interfaces and 29 from Tablet PCs during the experiment, and all participants had submissions via both interfaces. This suggests a high level of robustness for the paper interface. As a result, both instructor and students were able to focus on the Active Learning process, and not be distracted by issues with the technology.

We also found that the unfamiliar paper interface may have caused usability problems. For example, participant P1 complained "the pen didn't send what you wrote exactly". The log shows P1 mistook the "deselect" button for "deleting", and his personal doodling had been submitted accidentally, which may have led to his negative impression. To avoid such issues, more user training and/or stronger real time feedback are needed for the new paper interface.

Note that the number of activities was limited by the available time, as each activity involved a series of actions including question announcement, student thinking, answer collection, submission comments and discussion. Plus, given the small class size, the instructor commented on almost every student submission to encourage student participation.

\subsubsection{Interface Integration}

Here we consider the interface integration in terms of interface compatibility, user interaction and workflow. First, the paper interface is confirmed to be compatible with the original Tablet PC interface, because in the experiment both interfaces worked concurrently without interference. This is important to supporting heterogeneous device deployment as well as varying user preferences and needs.

Second, students could easily integrate paper interaction with the learning activities. They spread out and browsed the handouts on their desks, wrote answers with pens and submitted results on their paper. Participant P2 preferred the paper interface because of the high degree of control over the handouts, noting "Sometimes, we can 
not control slides using Tablet PC'. However, two other participants, P3 and P4 complained of "too much paper to flip through", indicating unexpected inconveniences of the new interface.

Looking further into the questionnaire comments and logs, we identify two main sources of this problem: 1) the one-slide-per-page layout forces students to flip paper for every slide. Given an average of about 30 slides per session, manual flipping was indeed distracting and required more user effort. 2) Unstapled paper slides require more effort for the students to keep things organized; the original intent was for flexible navigation and comparison. As a result, such negative effects counteract paper's advantages of quick browsing, convenient bi-manual manipulation and flexible spatial layout [26]. Several students suggested using paper slides only for the submission slides, so that they could enjoy the writing experience of paper but avoid annoying paper flipping. Printing multiple slides per page is another way to reduce the interference.

Finally, for the workflow, it is revealed that PaperCP has a potential drawback in out-of-class logistics: because the printing takes time, the instructor usually has to finalize the slides the day before classes at the latest. This may prevent lecturers from doing "last-minute" work, a flexibility which some like. Moreover, once the slides are printed out, it becomes hard to change or even add slides. These workflow problems could be solved by an optimized printing facility for Anoto-enabled handouts.

\subsubsection{Gesture Commands}

Here, we examine the effectiveness of gesture commands for the selective submission and deletion functions. In general, students seldom used the lasso to select partial notes for submission on either the paper or Tablet PC interfaces. The two interfaces each had only one user of the lasso selection. This phenomenon can be attributed to the design of the presentation slides: the submission slides are usually dedicated to the activity, so almost all user writing on those slides were answers for class activities; thus few lasso gestures were required to distinguish public notes from private ones.

The deletion gesture also suffered from infrequent use. This could be attributed to the weak feedback of the paper interface, which the users were not used to. The inconvenience of pen-switching is another possible reason. This suggests the importance of stronger feedback and single-pen operations.

\section{Future Work}

Our preliminary experiment has effectively proven the feasibility of the paper interface and its compatibility with existing practices. It has also revealed challenges in the design of the paper interface. In response, we will investigate appropriate layouts of printed slides. Specifically, we will consider factors such as the number of sheets of paper, the space for pen input, and the content of slides, as well as students' notetaking styles. This will help us to better understand the previously revealed problems and to validate proposed solutions.

Moreover, as the experiment analysis suggests, real-time feedback is the key for complex interactions on paper. It could be useful to enhance the current system with a pen-top feedback mechanism compatible with standard pen-and-paper interaction. For 
instance, the multimodal feedback pen [19] proposed by Liao et al. can provide realtime feedback information via built-in LEDs, vibration motors, and speech. Furthermore, considering users' high likelihood of working in a computer-rich environment, it will be interesting to combine the electronic and paper interfaces, e.g., providing digital visual feedback on a handheld device for paper interaction and incorporating multimedia data into student submissions. Similar methods are used in systems like Paper++ [23] and PaperLink [6], which employ a separate display or PDA to render dynamic content associated with specific regions in paper documents.

\section{Conclusion}

This paper examined the interplay between physical and digital affordances via a case study of an Active Learning support system. We identify key usability issues with the full digital system and present a paper-based interface which combines paper flexibility with the digital editing and communication. By deploying the system in a real classroom and conducting experiments in comparison with the Tablet PC interface, we have confirmed the feasibility of such a paper interface within a digital infrastructure. Our experiment also suggests that print layout, pen configuration, and sufficient user training are crucial aspects for paper interfaces to retain the tangibility advantage of the physical interface.

\section{Acknowledgements}

This work was supported by Microsoft Research (as part of the Microsoft Center for Interaction Design and Visualization at the University of Maryland) and NSF under Grant IIS-0414699 and IIS-00447730. We would like to thank all volunteer participants and appreciate Anoto for their support with streaming pattern space, Logitech for streaming software, and HP for hardware donations.

\section{References}

1. Abowd, G.D.: Classroom 2000: an experiment with the instrumentation of a living educational environment. IBM Syst. J. 38(4), 508-530 (1999)

2. Anderson, R., VanDeGrift, T., Wolfman, S.A., Yasuhara, K., Anderson, R.: Interaction patterns with a classroom feedback system: making time for feedback. In: Proceedings of CHI '03 extended abstracts, pp. 880-881 (2003)

3. Anderson, R., Anderson, R., Simon, B., Wolfman, S.A., VanDe Grift, T., Yasuhara, K.: Experiences with a tablet PC based lecture presentation system in computer science courses. In: Proceedings of the 35th SIGCSE technical symposium on computer science education, pp. 56-60

4. Angelo, T.A., Cross, K.P.: Classroom Assessment Techniques: A Handbook for College Teachers. Jossey-Bass Publishers, San Francisco (1993)

5. Anoto, Development Guide for Service Enabled by Anoto Functionality. Anoto (2002)

6. Arai, T., Aust, D., Hudson, S.E.: PaperLink: a technique for hyperlinking from real paper to electronic content. In: Proceedings of CHI'97, pp. 327-334 (1997)

7. Bligh, D.A.: What's the Use of Lectures? Jossey-Bass Publishers, San Francisco (2000) 
8. Bluegiga, Bluetooth Access Server, http://www.bluegiga.com/

9. Bransford, J.D., Brown, A.L., Cocking, R.R.e.: How People Learn: Brain, Mind, Experience, and School (Expanded Edition). National Academy Press, Washington (2000)

10. Dufresne, R., Gerace, W., Leonard, W., Mestre, J., Wenk, L.: Classtalk: A Classroom Communication System for Active Learning. Journal of Computing in Higher Education 7, 3-47 (1996)

11. DyKnow (2006), http://www.dyknow.com/

12. Greenberg, S., Boyle, M., LaBerge, J.: PDAs and shared public displays: Making personal information public, and public information personal. Personal Technologies 3(1) (1999)

13. Guimbretiere, F.: Paper Augmented Digital Documents. In: Proceedings of UIST'03, pp. 51-60 (2003)

14. Hinckley, K., Ramos, G., Guimbretiere, F., Baudisch, P., Smith, M.: Stitching: Pen Gestures that Span Multiple Displays. In: Proceedings of AVI'04, pp. 23-31 (2004)

15. Holman, D., Vertegaal, R., Altosaar, M., Troje, N., Johns, D.: Paper windows: interaction techniques for digital paper. In: Proceedings of CHI'05, pp. 591-599 (2005)

16. Johnson, D., Johnson, R., Smith, K.: Active Learning: Cooperation in the College Classroom. Interaction Book Company, Minnesota (1998)

17. Kam, M., Wang, J., Iles, A., Tse, E., Chiu, J., Glaser, D., Tarshish, O., Canny, J.: Livenotes: a system for cooperative and augmented note-taking in lectures. In: Proceedings of CHI'05, pp. 531-540 (2005)

18. Liao, C., Guimbretière, F., Hinckley, K.: PapierCraft: a command system for interactive paper. In: Proceedings of UIST05, pp. 241-244 (2005)

19. Liao, C., Guimbretière, F., Loeckenhoff, C.E.: Pentop feedback for paper-based interfaces. In: Proceedings of UIST'06, pp. 211-220 (2006)

20. Mackay, W.E., Pothier, G., Letondal, C., Bøegh, K., Sørensen, H.E.: The missing link: augmenting biology laboratory notebooks. In: Proceedings of UIST’02, pp. 41-50 (2002)

21. Mazur, E.: Peer Instruction: A User's Manual. Prentice Hall, New Jersey (1997)

22. Nelson, L., Ichimura, S., Pedersen, E.R., Adams, L.: Palette: a paper interface for giving presentations. In: Proceedings of CHI'99, pp. 354-361 (1999)

23. Norrie, M.C.,Signer, B.: Switching Over to Paper: A New Web Channel. In: Proceedings of Web Information Systems Engineering'03, pp. 209-218 (2003)

24. Ratto, M., Shapiro, R.B., Truong, T.M., Griswold, W.G.: The ActiveClass Project: Experiments in Encouraging Classroom Participation. In: Proceedings of CSCL'03, pp. 477$486(2003)$

25. Razmov, V.,, Anderson, R.: Pedagogical techniques supported by the use of student devices in teaching software engineering. In: Proceedings of SIGCSE'06, pp. 344-348 (2006)

26. Sellen, A.J., Harper, R.H.R.: The Myth of the Paperless Office, 1st edn. MIT press, Cambridge (2001)

27. Signer, B.: Fundamental Concepts for Interactive Paper and Cross-Media Information Spaces ( ETH No. 16218), PhD thesis, Swiss Federal Institute of Technology, Zu$\operatorname{rich}(2005)$

28. Wellner, P.: Interacting with paper on the DigitalDesk. Communications of the ACM 36(7), 87-96 (1993)

29. Wilkerson, M., Griswold, W.G., Simon, B.: Ubiquitous presenter: increasing student access and control in a digital lecturing environment. In: Proceedings of SIGCSE'05, pp. 116-120 (2005)

30. Yeh, R.B., Liao, C., Klemmer, S.R., Guimbretière, F., Lee, B., Kakaradov, B., Stamberger, J., Paepcke, A.: ButterflyNet: A Mobile Capture and Access System for Field Biology Research. In: Proceedings of CHI'06, pp. 571-580 (2006) 